\title{
The safety of excimer laser as an alternative to classical, balloon angioplasty methods
}

\author{
Authors: Piotr Machowiec ${ }^{1}$, Gabriela Ręka ${ }^{1}$, Halina Piecewicz-Szczęsna ${ }^{2}$ (mentor) \\ ${ }^{1}$ Students' Scientific Association of Chair and Department of Epidemiology and Clinical Research \\ Methodology, Medical University of Lublin, Poland \\ ${ }^{2}$ Chair and Department of Epidemiology and Clinical Research Methodology, Medical University of \\ Lublin, Poland
}

DOI: https://doi.org/10.26800/LV-142-supp5-62

\section{Introduction:}

The procedure of laser atherectomy is a long-established adjunctive therapy in percutaneous vascular interventions. Initial studies were not promising due to similar or lower efficacy compared to conventional angioplasty and greater complication rate. However, the introduction of new, low-profile catheters with high parameters of transmitted energy, a change in the procedure for performing angioplasty and the proper qualification of the lesions resulted in a reduction of adverse effects.

\section{Aim:}

The study aims to present the current state of knowledge concerning the usage of excimer lasers in coronary and peripheral artery stenosis as well as its safety aspect.

\section{Material and methods:}

The article is a review of 10 recent publications available on the PubMed and Google Scholar databases since 2015. They were picked after performing the analysis using the keywords linked by logical operators: "excimer laser" and "angioplasty".

\section{Results:}

The main indications for the use of the laser include: ostial lesions, long, calcified lesions $>20 \mathrm{~mm}$ and moderately and severely calcified lesions. Excimer laser coronary atherectomy (ELCA) is also a safe technique for the treatment of in-stent restenosis, and is associated with a relatively low recurrent restenosis in comparison with scoring balloon dilatation alone. Nevertheless, the use of the ELCA procedure involves a certain risk of complications of which vessel perforation is the worst adverse event, but it occurs seldom. Furthermore, there are isolated reports describing the use of ELCA in the treatment of acute coronary syndromes and peripheral artery disease with good clinical outcome and with only minor side effects reported.

\section{Conclusion:}

The excimer laser is a safe and feasible alternative to classic treatment methods, mainly balloon angioplasty, but the further research assessing the safety of this procedure is required.

Keywords: angioplasty, atherosclerosis, excimer laser 